\title{
Exponential Convergence of an Observer Based on Partial Field Measurements for the Wave Equation
}

\author{
Dominique Chapelle, Nicolae Cîndea, \\ Maya De Buhan, and Philippe Moireau
}

\author{
MACS Project Team, Inria, France \\ Correspondence should be addressed to Philippe Moireau, philippe.moireau@inria.fr
}

Received 27 July 2012; Accepted 2 October 2012

Academic Editor: Valery Yakhno

Copyright (C) 2012 Dominique Chapelle et al. This is an open access article distributed under the Creative Commons Attribution License, which permits unrestricted use, distribution, and reproduction in any medium, provided the original work is properly cited.

We analyze an observer strategy based on partial—that is, in a subdomain-measurements of the solution of a wave equation, in order to compensate for uncertain initial conditions. We prove the exponential convergence of this observer under a nonstandard observability condition, whereas using measurements of the time derivative of the solution would lead to a standard observability condition arising in stabilization and exact controlabillity. Nevertheless, we directly relate our specific observability condition to the classical geometric control condition. Finally, we provide some numerical illustrations of the effectiveness of the approach.

\section{Introduction}

Observer theory has been established for decades [1], but only much more recently has it been considered for systems governed by evolution partial differential equations (PDEs), see [2-4] and references therein. In this realm challenges abound, as in particular observer convergence can no longer be mathematically analyzed solely by investigating the poles of the observer dynamics operator. Furthermore, observers are only meaningful when they provide an actual computable estimate of the system state-and possibly also of some uncertain parameters, see [2] — which implies that we must consider discretization issues both in their design and in their analysis, and this in turn introduces additional serious difficulties, as is already well known in the field of stabilization of PDE-governed systems, see for example [5].

In this paper we consider an observer strategy originally proposed in [6] under the name "Schur Displacement Feedback" (SDF) for elasticity-like formulations, and directly adapted here to the scalar wave equation. A major advantage of this observer is that it exploits measurements of the primary variable rather than of the time derivative of this 
variable. Such direct measurements are more easily obtained in practice, in general, hence no detrimental time differentiation of the data is required in this case. When analyzing the estimation error, we find-as always with Luenberger observers-a closed-loop stabilized dynamics, albeit here of a rather uncommon-nonphysical-type. Nevertheless, we will show that exponential convergence is achieved under an observability condition closely related to that associated with more standard stabilization strategies, a condition which is itself equivalent to the classical geometric control condition [7].

\section{Observer Design}

Let $\Omega$ be a bounded domain of $\mathbb{R}^{n}$ with a regular boundary $\partial \Omega$. Given a known source term $f \in L^{1}\left(\mathbb{R}^{+}, L^{2}(\Omega)\right)$ with $\dot{f}$-namely, the time derivative of $f$-also in $L^{1}\left(\mathbb{R}^{+}, L^{2}(\Omega)\right)$, we consider the following wave equation:

$$
\begin{gathered}
\ddot{u}(x, t)-\Delta u(x, t)=f(x, t), \quad(x, t) \in \Omega \times(0, \infty), \\
u(x, t)=0, \quad(x, t) \in \partial \Omega \times(0, \infty), \\
u(x, 0)=u_{0}(x), \quad \dot{u}(x, 0)=v_{0}(x), \quad x \in \Omega .
\end{gathered}
$$

Denoting $x(t)=\left(\begin{array}{c}u(t) \\ \dot{u}(t)\end{array}\right)$, we can rewrite (2.1) as the first-order system

$$
\begin{gathered}
\dot{x}(t)=A x(t)+R, \quad t>0, \\
x(0)=x_{0},
\end{gathered}
$$

where $x_{0}=\left(\begin{array}{c}u_{0} \\ v_{0}\end{array}\right)$ and $A: \mathscr{\Phi}(A) \rightarrow \chi x$ with

$$
\Phi(A)=\Phi(-\Delta) \times \Phi\left(-\Delta^{1 / 2}\right), \quad X=\Phi\left(-\Delta^{1 / 2}\right) \times \mathscr{H}, \quad A=\left(\begin{array}{cc}
0 & I \\
\Delta & 0
\end{array}\right), \quad R=\left(\begin{array}{l}
0 \\
f
\end{array}\right),
$$

for $\mathscr{t}=L^{2}(\Omega)$ here. Note that with the boundary conditions considered in (2.1), we have $\Phi\left(-\Delta^{1 / 2}\right)=H_{0}^{1}(\Omega)$ and $\Phi(-\Delta)=H^{2}(\Omega) \cap H_{0}^{1}(\Omega)$. The operator $A$ generates a group and for all $x_{0} \in \mathcal{X}$ then $(u, \dot{u}) \in C((0, T) ; \mathcal{X})$ for any $T>0$, see for example [8].

For this system, we consider that some measurements are available-assumed to be without noise in this paper-in $\omega \subset \Omega$ an open and nonempty subset of $\Omega$ and at every time $t$. These measurements can be either of the form

$$
\forall t \geq 0, \quad \begin{array}{lc}
H_{0}^{1}(\Omega) & \longrightarrow H^{1}(\omega) \\
u(\cdot, t) & \longmapsto z(t)=\left.u(\cdot, t)\right|_{\omega}
\end{array}
$$

or alternatively

$$
\forall t \geq 0, \quad \mid \begin{aligned}
& L^{2}(\Omega) \quad \longrightarrow L^{2}(\omega) \\
& \dot{u}(\cdot, t) \longmapsto z(t)=\left.\dot{u}(\cdot, t)\right|_{\omega} .
\end{aligned}
$$


Our paper will mainly concentrate on the first type of observation, but we will consider the second type as a matter of comparison. In each case, accordingly introducing the observation space $\mathfrak{Z}=H^{1}(\omega)$ or $\mathfrak{Z}=L^{2}(\omega)$, we can define an observation operator $H \in \mathcal{L}(\mathcal{X}, \mathfrak{Z})$ by

$$
H=\left(\begin{array}{ll}
H_{0} & 0
\end{array}\right), \quad \text { or } \quad H=\left(\begin{array}{ll}
0 & H_{0}
\end{array}\right)
$$

respectively, with $H_{0}$ the restriction operator on $\omega$.

The aim in observer design is to define a system $\hat{x}$, with modified dynamics compared to that followed by the original system $x$ and based on using the discrepancy $z-H \hat{x}$, so that for any initial condition $\widehat{x}_{0}$, the state system $\widehat{x}$ tends to $x$ in time. The dynamics of the observer $\hat{x}$ read

$$
\begin{gathered}
\dot{\hat{x}}(t)=A \widehat{x}(t)+R+G(z(t)-H \widehat{x}(t)), \quad t>0, \\
\widehat{x}(0)=\widehat{x}_{0},
\end{gathered}
$$

with $G$ a gain operator to be defined in $\mathcal{L}(\mathfrak{Z}, \mathcal{X})$ in our case.

In order to assess the efficiency of the observer, we can consider the dynamics followed by the error $\tilde{x}=x-\widehat{x}$, namely

$$
\begin{gathered}
\dot{\tilde{x}}=(A-G H) \tilde{x}(t), \quad t>0, \\
\tilde{x}(0)=x_{0}-\widehat{x}_{0},
\end{gathered}
$$

and then $G$ should stabilize $\tilde{x}$ to 0 . This type of approach can be categorized as a Luenberger observer [1] —also sometimes referred to as "nudging" [3] —applied on an evolution PDE.

In the case of time-derivative measurements (2.5), we can choose $G=\gamma\left(\underset{\mathbb{1}_{\omega}}{0}\right)$, for any $\gamma>0$. Then the observer system derived from a Direct Velocity Feedback (DVF) gives

$$
\begin{gathered}
\ddot{\widehat{u}}(x, t)-\Delta \widehat{u}(x, t)=f+\gamma \mathbb{1}_{\omega}(x)(z(x, t)-\dot{\widehat{u}}(x, t)), \quad(x, t) \in \Omega \times(0, \infty), \\
\widehat{u}(x, t)=0, \quad(x, t) \in \partial \Omega \times(0, \infty), \\
\widehat{u}(x, 0)=\widehat{u}_{0}(x), \quad \dot{\hat{u}}(x, 0)=\widehat{v}_{0}(x), \quad x \in \Omega,
\end{gathered}
$$

and the error follows the dynamics

$$
\begin{gathered}
\ddot{\tilde{u}}(x, t)-\Delta \tilde{\tilde{u}}(x, t)+\gamma \mathbb{1}_{\omega}(x) \dot{\tilde{u}}(x, t)=0, \quad(x, t) \in \Omega \times(0, \infty), \\
\tilde{u}(x, t)=0, \quad(x, t) \in \partial \Omega \times(0, \infty), \\
\tilde{u}(x, 0)=\tilde{u}_{0}(x), \quad \dot{\tilde{u}}(x, 0)=\widetilde{v}_{0}(x), \quad x \in \Omega .
\end{gathered}
$$


This system is well known to be exponentially stable—see [7, 9] and references therein-if and only if the observability condition

$$
\begin{aligned}
& \exists\left(T_{0}, C\right), \quad \forall T \geq T_{0}, \quad \forall\left(u_{0}, v_{0}\right) \in H_{0}^{1}(\Omega) \times L^{2}(\Omega), \\
& \int_{0}^{T} \int_{\omega}|\dot{u}(x, t)|^{2} \mathrm{~d} \Omega \mathrm{d} t \geq C\left(\left\|u_{0}\right\|_{H^{1}(\Omega)}^{2}+\left\|v_{0}\right\|_{L^{2}(\Omega)}^{2}\right),
\end{aligned}
$$

is satisfied for arbitrary solutions of the system (2.1) with $f=0$, which is equivalent to the geometric control condition (GCC) of [7].

In the first case of direct measurements of the field (2.4), we can also propose an observer without any time differentiation involved-typically to avoid amplifying the measurement errors which always arise in practice. To that purpose, we define the operator

$$
\left\llcorner_{\omega}: H^{1}(\omega) \longrightarrow H_{0}^{1}(\Omega), \quad\left\llcorner_{\omega} \phi=\psi,\right.\right.
$$

where $\psi$ is the solution of the following elliptic equation:

$$
\begin{array}{cc}
\Delta \psi=0, & \text { in } \Omega \backslash \omega, \\
\psi=0, & \text { on } \partial \Omega, \\
\psi=\phi, & \text { in } \bar{\omega},
\end{array}
$$

namely, $\mathcal{L}_{\omega}$ is a harmonic lifting operator. We easily verify that $\mathcal{L}_{\omega}$ is bounded from $H^{1}(\omega)$ to $H_{0}^{1}(\Omega)$ using the trace and lifting properties to write

$$
\left\|\mathcal{L}_{\omega} \phi\right\|_{H_{0}^{1}(\Omega)}^{2} \leq\|\nabla \phi\|_{L^{2}(\omega)}^{2}+C_{1}\left\|\left.\phi\right|_{\partial \omega}\right\|_{H^{1 / 2}(\partial \omega)}^{2} \leq C_{2}\|\phi\|_{H^{1}(\omega)}^{2}
$$

As a candidate observer, we will consider $G=\gamma\left(\begin{array}{c}\mathcal{L}_{\omega} \\ 0\end{array}\right)$ which gives the following first-order system:

$$
\begin{gathered}
\dot{\hat{u}}(x, t)=\widehat{v}(x, t)+\gamma \mathcal{L}_{\omega}\left(z(t)-\left.\mathbb{1}_{\omega}(x) \widehat{u}(x, t)\right|_{\omega}\right), \quad(x, t) \in \Omega \times(0, \infty), \\
\dot{\hat{v}}(x, t)-\Delta \widehat{u}(x, t)=0, \quad(x, t) \in \Omega \times(0, \infty), \\
\widehat{u}(x, 0)=\widehat{u}_{0}(x), \quad \widehat{v}(x, 0)=\widehat{v}_{0}(x), \quad x \in \Omega
\end{gathered}
$$

with $\gamma>0$ a gain parameter. Note that this system amounts to a modification of the wave equation written in first-order form, with a correction term based on the discrepancy between the measurement and the observer primary variable. This strategy is the direct adaptation to the wave equation of the "Schur Displacement Feedback" (SDF) filtering methodology originally proposed in [6] for elasticity-like formulations. 
Remark 2.1. The above choices of gain operator $G$ fulfill the identity $G=\gamma H^{*}$ where the * symbol denotes the adjoint via Riesz representation. This is obvious in the case of (2.5), whereas we will provide the detailed proof with Proposition 3.1 below for (2.4).

Remark 2.2. We consider in this paper a known source term, but the observers considered here can be extended to also estimate an unknown source term, following the strategy introduced in [2].

\section{Exponential Convergence of the Observer System for Field Measurements}

In order to establish that (2.15) is an adequate observer for (2.1), we should study the decay of the error satisfying for (2.15) the following system:

$$
\begin{gathered}
\dot{\tilde{u}}(x, t)=\tilde{v}(x, t)-\gamma \mathcal{L}_{\omega}\left(\mathbb{1}_{\omega}(x) \tilde{u}(x, t)\right), \quad(x, t) \in \Omega \times(0, \infty), \\
\dot{\tilde{v}}(x, t)-\Delta \tilde{u}(x, t)=0, \quad(x, t) \in \Omega \times(0, \infty), \\
\tilde{u}(x, t)=0, \quad(x, t) \in \partial \Omega \times(0, \infty), \\
\tilde{u}(x, 0)=\tilde{u}_{0}(x), \quad \tilde{v}(x, 0)=\widetilde{v}_{0}(x), \quad x \in \Omega
\end{gathered}
$$

and we will prove the exponential decay of the associated energy, namely,

$$
\widetilde{E}(t)=\frac{1}{2}\left(\int_{\Omega}|\nabla \tilde{u}(x, t)|^{2} \mathrm{~d} \Omega+\int_{\Omega}|\widetilde{v}(x, t)|^{2} \mathrm{~d} \Omega\right) .
$$

Proposition 3.1. Assume that we have the observability condition

$$
\int_{0}^{T_{0}}\|u(\cdot, t)\|_{H^{1}(\omega)}^{2} d t \geq C\left(\left\|u_{0}\right\|_{H^{1}(\Omega)}^{2}+\left\|v_{0}\right\|_{L^{2}(\Omega)}^{2}\right), \quad \forall\left(u_{0}, v_{0}\right) \in H_{0}^{1}(\Omega) \times L^{2}(\Omega)
$$

for some $T_{0}>0$, for arbitrary solutions of (2.1) with $f=0$. Then, there exist strictly positive constants $M$ and $\mu$ such that

$$
\widetilde{E}(t) \leq M e^{-\mu t} \widetilde{E}(0), \quad \forall t>0 .
$$


Proof. Let us consider the space $H_{\omega}^{1}$ defined by $H^{1}(\omega)$ equipped with the norm

$$
\|\phi\|_{H_{\omega}^{1}}=\left\|\nabla\left(\mathcal{L}_{\omega} \phi\right)\right\|_{L^{2}(\Omega)}
$$

It is straightforward to see that this norm is equivalent to the usual norm of $H^{1}(\omega)$, using first (2.14), and secondly

$$
\begin{aligned}
\|\phi\|_{H^{1}(\omega)}^{2} & =\|\nabla \phi\|_{L^{2}(\omega)}^{2}+\|\phi\|_{L^{2}(\omega)}^{2} \\
& \leq\left\|\nabla\left(\mathcal{L}_{\omega} \phi\right)\right\|_{L^{2}(\Omega)}^{2}+\left\|\mathcal{L}_{\omega} \phi\right\|_{L^{2}(\Omega)}^{2} \leq(1+C)\left\|\nabla\left(\mathcal{L}_{\omega} \phi\right)\right\|_{L^{2}(\Omega)^{\prime}}^{2}
\end{aligned}
$$

with $C$ given by the Poincaré inequality. Considering now $\mathcal{L}_{\omega}$ as a bounded operator from $H_{\omega}^{1}$ to $H_{0}^{1}(\Omega)$, we want to identify $\mathcal{L}_{\omega}^{*}$. Using the orthogonality property

$$
\forall \psi \in H_{0}^{1}(\Omega) \text { s.t. } \psi \mid \omega=0, \quad\left\langle\nabla\left(\mathcal{L}_{\omega} \phi\right), \nabla \psi\right\rangle_{L^{2}(\Omega)}=0,
$$

directly inferred from the first equation of (2.13), we have that for all $\psi \in H_{0}^{1}(\Omega)$ and $\phi \in H_{\omega}^{1}$

$$
\left\langle\nabla\left(\mathcal{L}_{\omega} \phi\right), \nabla \psi\right\rangle_{L^{2}(\Omega)}=\left\langle\nabla\left(\mathcal{L}_{\omega} \phi\right), \nabla\left(\mathcal{L}_{\omega} \psi \mid \omega\right)\right\rangle_{L^{2}(\Omega)}=\langle\phi, \psi \mid \omega\rangle_{H_{\omega}^{1}}
$$

showing that the adjoint via Riesz representation is then

$$
\mathcal{L}_{\omega}^{*}: H_{0}^{1}(\Omega) \longrightarrow H_{\omega}^{1}, \quad \mathcal{L}_{\omega}^{*} \psi=\left.\psi\right|_{\omega}
$$

Hence, we have proven that $G=\gamma H^{*}$, as claimed in Remark 2.1. Therefore, the error system (3.1) dynamics can be rewritten as

$$
\dot{\tilde{x}}=\left(A-\gamma H^{*} H\right) \tilde{x}(t)
$$

and the exponential decay of the energy (3.2) is then equivalent to the following observability inequality, see for example Theorem 2.3 in [9] with $B=H^{*}$,

$$
\int_{0}^{T_{0}}\|H x\|_{H_{\omega}^{1}}^{2} \mathrm{~d} t \geq C\left\|x_{0}\right\|_{x^{\prime}}^{2}, \quad \forall x_{0} \in \mathcal{X},
$$

where $x$ is the solution of the original wave equation in first-order form (2.2) with $f=0$, and for some strictly positive constants $T_{0}$ and $C$, and of course this condition is directly equivalent to (3.3). 
However, the observability condition (3.3) is somewhat nonstandard since we are using the $H^{1}$-norm of the field in the observation space instead of the $L^{2}$-norm of the time derivative. Nevertheless, the following proposition directly relates our observability condition with classical results.

Proposition 3.2. Assume that the geometric control condition of Bardos et al. [7] is satisfied for some $\breve{\omega}$ strict subset of $\omega$ such that $\operatorname{dist}(\Omega \backslash \omega, \breve{\omega})>0$, in the time interval $] 0, T_{0}\left[\right.$ for $T_{0}>0$. Then the observability condition (3.3) holds for the same time $T_{0}$.

Proof. Since the geometric control condition holds, we have the classical observability condition [7]

$$
\int_{0}^{\breve{T}} \int_{\breve{\omega}}|\dot{u}(x, t)|^{2} \mathrm{~d} \Omega \mathrm{d} t \geq C\left(\left\|u_{0}\right\|_{H^{1}(\Omega)}^{2}+\left\|v_{0}\right\|_{L^{2}(\Omega)}^{2}\right), \quad \forall\left(u_{0}, v_{0}\right) \in H_{0}^{1}(\Omega) \times L^{2}(\Omega),
$$

for some time $\breve{T}=T_{0}-\delta$ with $\delta$ sufficiently small, for arbitrary solutions of (2.1) with $f=0$. We will show that this entails (3.3) by an argument inspired from [10]. Let $\psi \in C_{c}^{\infty}(\bar{\Omega})$ be a cutoff function satisfying

$$
\psi(x)= \begin{cases}0, & \text { if } x \in \Omega \backslash \omega \\ 1, & \text { if } x \in \breve{\omega}\end{cases}
$$

and $0 \leq \psi(x) \leq 1$ for every $x \in \bar{\Omega}$. Denote also $\phi(t)=t^{2}(\breve{T}-t)^{2}$. Then, by repeated integrations by parts, we obtain

$$
\begin{aligned}
0= & \int_{0}^{\breve{T}} \int_{\omega} \phi \psi(\ddot{u}-\Delta u) u \mathrm{~d} \Omega \mathrm{d} t \\
= & \int_{0}^{\breve{T}} \int_{\omega} \ddot{\phi} \psi \frac{|u|^{2}}{2} \mathrm{~d} \Omega \mathrm{d} t-\int_{0}^{\breve{T}} \int_{\omega} \phi \psi|\dot{u}|^{2} \mathrm{~d} \Omega \mathrm{d} t+\int_{0}^{\breve{T}} \int_{\partial \omega} \phi \frac{\partial \psi}{\partial n} \frac{|u|^{2}}{2} \mathrm{~d} \Gamma \mathrm{d} t \\
& -\int_{0}^{\breve{T}} \int_{\omega} \phi \Delta \psi \frac{|u|^{2}}{2} \mathrm{~d} \Omega \mathrm{d} t+\int_{0}^{\breve{T}} \int_{\omega} \phi \psi|\nabla u|^{2} \mathrm{~d} \Omega \mathrm{d} t .
\end{aligned}
$$

The definition of $\psi$ entails $\left.(\partial \psi / \partial n)\right|_{\partial \omega}=0$, hence

$$
\int_{0}^{\breve{T}} \int_{\omega} \phi \psi|\dot{u}|^{2} \mathrm{~d} \Omega \mathrm{d} t=\int_{0}^{\breve{T}} \int_{\omega} \phi \psi|\nabla u|^{2} \mathrm{~d} \Omega \mathrm{d} t+\int_{0}^{\breve{T}} \int_{\omega} \ddot{\phi} \psi \frac{|u|^{2}}{2} \mathrm{~d} \Omega \mathrm{d} t-\int_{0}^{\breve{T}} \int_{\omega} \phi \Delta \psi \frac{|u|^{2}}{2} \mathrm{~d} \Omega \mathrm{d} t .
$$


This identity combined with the properties of the cutoff functions $\phi$ and $\psi$ provides, for any strictly positive $\varepsilon$, the existence of a constant $C>0$ such that

$$
\int_{\varepsilon}^{\breve{T}-\varepsilon} \int_{\breve{\omega}}|\dot{u}|^{2} \mathrm{~d} \Omega \mathrm{d} t \leq C\left(\int_{0}^{\breve{T}} \int_{\omega}|\nabla u|^{2} \mathrm{~d} \Omega \mathrm{d} t+\int_{0}^{T} \int_{\omega}|u|^{2} \mathrm{~d} \Omega \mathrm{d} t\right)
$$

Substituting $\breve{T}+2 \varepsilon$ for $\breve{T}$ in all the above computations gives

$$
\int_{\varepsilon}^{\breve{T}+\varepsilon} \int_{\breve{\omega}}|\dot{u}|^{2} \mathrm{~d} \Omega \mathrm{d} t \leq C \int_{0}^{\breve{T}+2 \varepsilon}\|u(\cdot, t)\|_{H^{1}(\omega)}^{2} \mathrm{~d} t .
$$

We proceed by making the change of variable $\tau=t-\varepsilon$ in the left-hand side integral, yielding

$$
\int_{0}^{\breve{T}} \int_{\breve{\omega}}|\dot{u}(x, \tau+\varepsilon)|^{2} \mathrm{~d} \Omega \mathrm{d} \tau \leq C \int_{0}^{\breve{T}+2 \varepsilon}\|u\|_{H^{1}(\omega)}^{2} \mathrm{~d} t
$$

Noting that $u(x, t+\varepsilon)$ satisfies the wave equation with initial data $(u(x, \varepsilon), \dot{u}(x, \varepsilon))$ and applying (3.12) with this shifted solution, we obtain that there exists also $C$ such that

$$
\int_{0}^{\breve{T}} \int_{\breve{\omega}}|\dot{u}(x, t+\varepsilon)|^{2} \mathrm{~d} \Omega \mathrm{d} t \geq C\left(\|u(\varepsilon)\|_{H^{1}(\Omega)}^{2}+\|\dot{u}(\varepsilon)\|_{L^{2}(\Omega)}^{2}\right) .
$$

Combining (3.18), (3.19), and the the fact that the energy of the solution of the wave equation is exactly conserved over time, we have our observability inequality (3.3) upon choosing $\varepsilon=\delta / 2$.

Remark 3.3. Note that this proof requires a geometric control condition slightly stronger than that directly associated with the measurement domain $\omega$, since $\breve{\omega}$ is a strict subset. However, it is likely that our desired observability condition (2.11) could also be established directly by adapting a microlocal analysis strategy used for the standard observability condition (3.3), for example, using defect measures [11]. In our case, the defect measure of interest would be in $H^{1}$ as considered more recently in [12]. This $H^{1}$ defect measure should be proven to be made of the same bicharacteristic set as the $L^{2}$ defect measure introduced in [13] for the classical GCC. In this context we would have the new observability condition with the same subdomain $\omega$. Nevertheless, the advantage of our approach lies in its compactness, and moreover it demonstrates an interesting property of equirepartition (over time) of the total energy localized within the subdomain $\omega$ between the kinetic and potential contributions. 


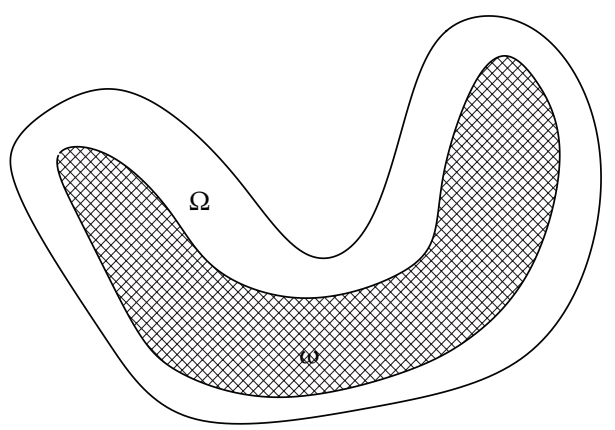

(a) Large observation domain

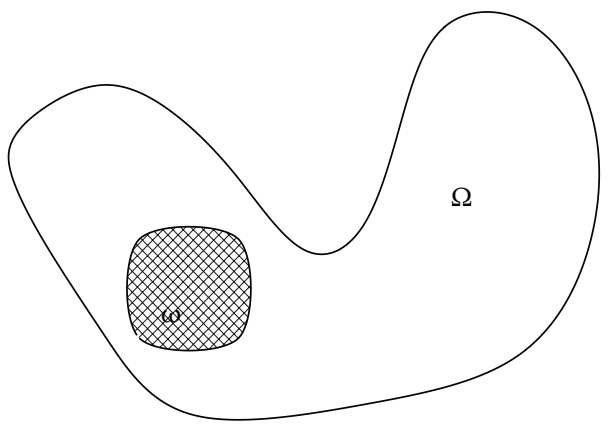

(b) Small observation domain

Figure 1: Geometry and two observation domains considered.

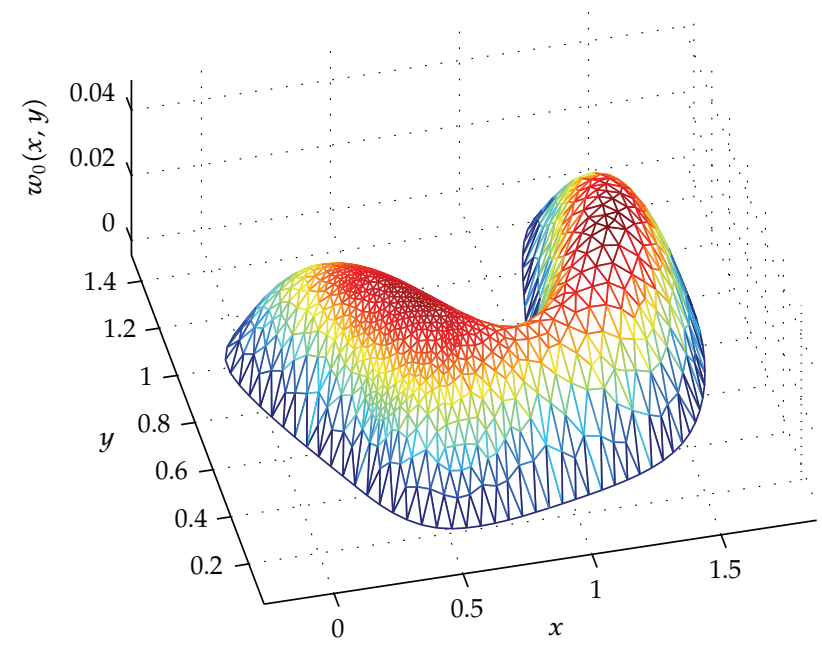

Figure 2: Initial condition in mesh used.

Remark 3.4. For both observer strategies, the potential noise in the measurementsdisregarded in this paper-would simply entail an additional source term in the error equations, without amplification of this error term by time or space differentiation. The parameter $\gamma$ should then be chosen to obtain the best decay rate without undue amplification of the noise $[2,6]$.

\section{Numerical Illustrations}

In order to illustrate the effectiveness of our Schur Displacement Feedback (SDF) observer approach-and compare it to the classical Direct Velocity Feedback (DVF) observer-we consider the two-dimensional domain shown in Figure 1 and, with the two choices of observation domains also displayed in the Figure. In order for the mesh to be conforming with the observation domain, we use one specific triangular mesh for each observation choice, with very similar mesh sizes-corresponding to about 950 vertices-for both cases, one of which being shown in Figure 2 with the initial condition considered in the simulations. 


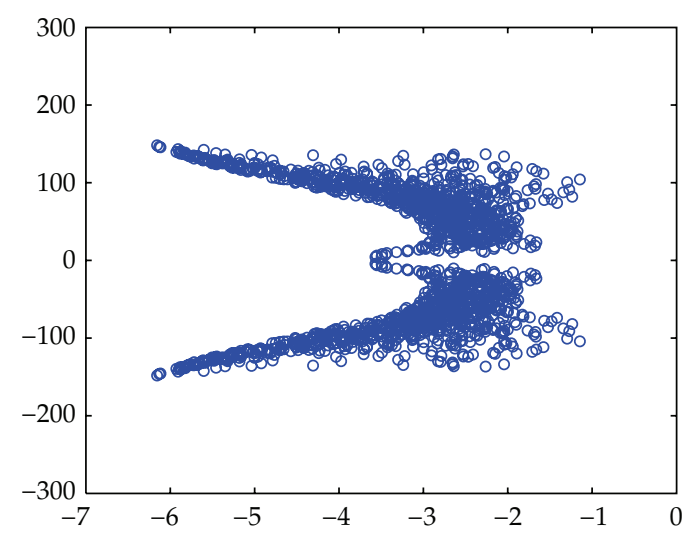

(a) SDF-large observation domain

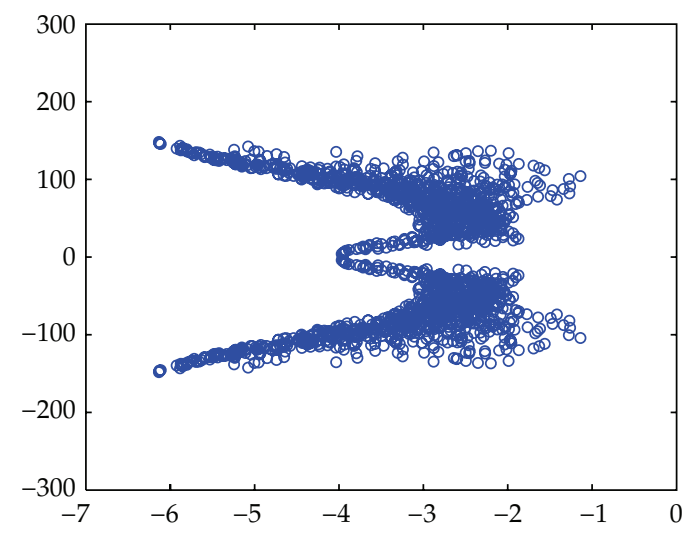

(c) DVF-large observation domain

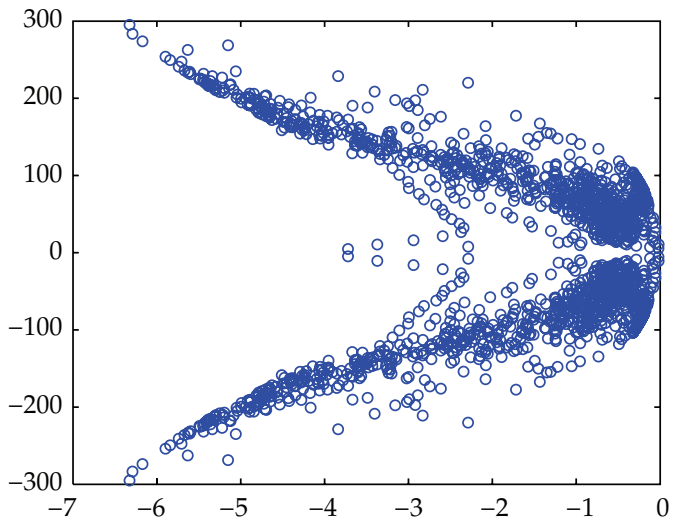

(b) SDF—small observation domain

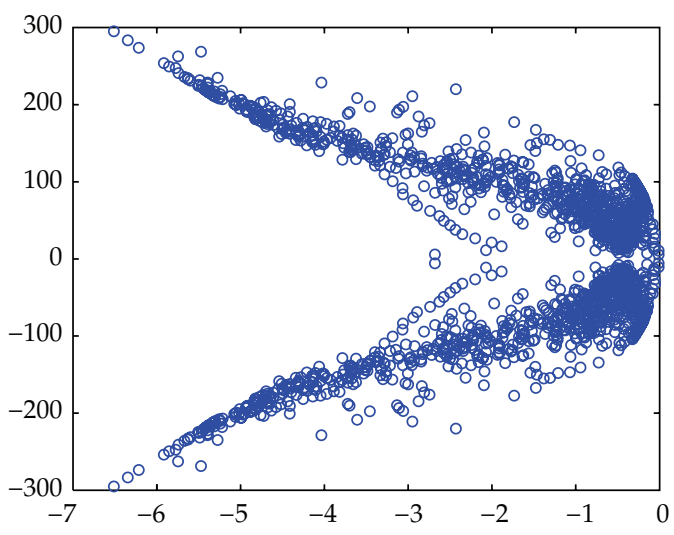

(d) DVF-small observation domain

Figure 3: Poles for SDF and DVF stabilization (up and down, resp.) with large and small observation domains (left and right, resp.).

The variational form of the wave equation is then discretized using $P_{1}$-Lagrange finite elements in space and a mid-point Newmark type time-discretization scheme [6]. As discretization issues in relation to observation and stabilization properties are not the topic of this paper-we instead refer to $[6,14]$ for detailed discretization considerations and analysis-we use sufficiently small values of the time step to obtain "converged" solutions in time, and we focus on the semidiscrete equation-namely, discretized space-wise onlywhen computing poles. Furthermore, as it is well known that spatial discretization itself induces undesirable numerical artifacts in the stabilization and control of our type of partial differential equation, with some numerical "high-frequency" poles featuring vanishing dissipation, we resort to incorporating a numerical viscosity term as advocated in $[14,15]$ to circumvent this difficulty.

We show in Figures 3(a) and 3(b) the numerical poles of the SDF-stabilized equation, for the two observation domains considered $(\gamma=8)$. We can see that the stabilization strategy is very effective for the large observation domain, as all real parts lie below -1 , and below -2 or so when excluding high-frequency poles. As a matter of comparison, 


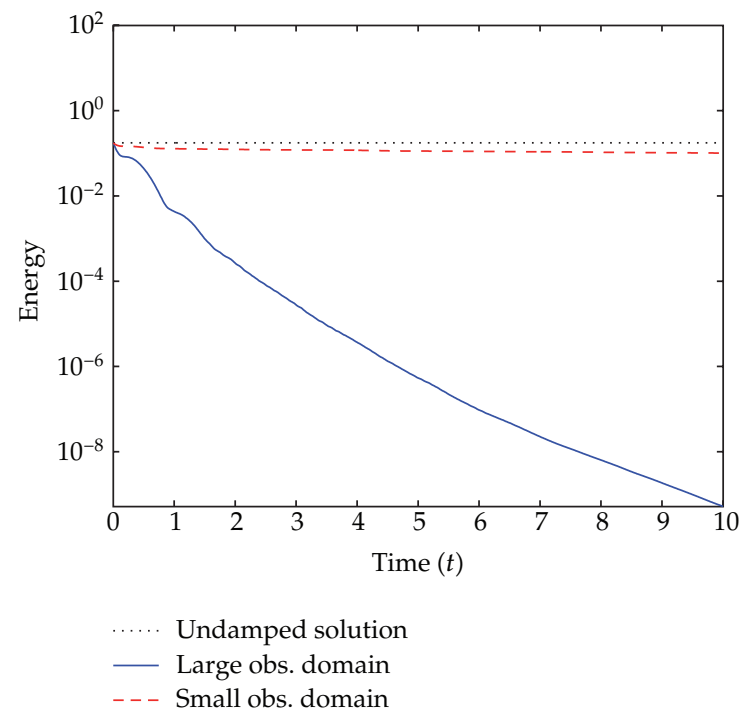

Figure 4: Energies of the undamped and SDF-stabilized solutions.

the imaginary part of the first undamped eigenmode is about 5.6. Of course, the effectiveness is significantly reduced in the case of the second observation domain, which does not satisfy the above geometric control condition, indeed. We also compare in Figures 3(c) and 3(d) the corresponding poles obtained with the DVF strategy $(\gamma=8)$, and we observe that the two approaches provide similar stabilization properties.

This is further illustrated in Figure 4, where we compare the energy norms of the SDF-stabilized numerical solutions in time with that of the wave equation itself-note that the time discretization scheme used is energy preserving. This confirms the excellent stabilization properties of the SDF approach, as expected provided that the controllability (or equivalent observability) condition is fulfilled. Of course, this directly translates into equivalent observation convergence properties following our above discussion.

\section{References}

[1] D. G. Luenberger, "An introduction to observers," IEEE Transactions on Automatic Control, vol. 16, no. 6, pp. 596-602, 1971.

[2] P. Moireau, D. Chapelle, and P. Le Tallec, "Joint state and parameter estimation for distributed mechanical systems," Computer Methods in Applied Mechanics and Engineering, vol. 197, no. 6-8, pp. 659-677, 2008.

[3] D. Auroux and J. Blum, "A nudging-based data assimilation method: the back and forth nudging (BFN) algorithm," Nonlinear Processes Geophysics, vol. 15, no. 2, pp. 305-319, 2008.

[4] K. Ramdani, M. Tucsnak, and G. Weiss, "Recovering the initial state of an infinite-dimensional system using observers," Automatica, vol. 46, no. 10, pp. 1616-1625, 2010.

[5] L. R. Tcheugoué Tébou and E. Zuazua, "Uniform exponential long time decay for the space semidiscretization of a locally damped wave equation via an artificial numerical viscosity," Numerische Mathematik, vol. 95, no. 3, pp. 563-598, 2003.

[6] P. Moireau, D. Chapelle, and P. Le Tallec, "Filtering for distributed mechanical systems using position measurements: perspectives in medical imaging," Inverse Problems, vol. 25, no. 3, Article ID 035010, 25 pages, 2009. 
[7] C. Bardos, G. Lebeau, and J. Rauch, "Un exemple d'utilisation des notions de propagation pour le contrôle et la stabilisation des problèmes hyperboliques," Rendiconti del Seminario Matematico del Universita Politecnico Torino, Fascicolo Speciale (Hyperbolic Equations), pp. 12-31, 1988.

[8] A. Pazy, Semigroups of Linear Operators and Applications to Partial Differential Equations, vol. 44 of Applied Mathematical Sciences, Springer, New York, NY, USA, 1983.

[9] K. Liu, "Locally distributed control and damping for the conservative systems," SIAM Journal on Control and Optimization, vol. 35, no. 5, pp. 1574-1590, 1997.

[10] X. Zhang, "Explicit observability estimate for the wave equation with potential and its application," Proceedings: Mathematical, Physical and Engineering Sciences, vol. 456, no. 1997, pp. 1101-1115, 2000.

[11] P. Gérard, "Microlocal defect measures," Communications in Partial Differential Equations, vol. 16, no. 11, pp. 1761-1794, 1991.

[12] N. Burq and G. Lebeau, "Mesures de défaut de compacité, application au système de lamé," Annales Scientifiques de l'École Normale Supérieure, vol. 34, no. 6, pp. 817-870, 2001.

[13] N. Burq and P. Gérard, Contrôle optimal des équations aux derivées partielles, Cours de l'Ecole Polytechnique, 2002.

[14] D. Chapelle, N. Cîndea, and P. Moireau, "Improving convergence in numerical analysis using observers-the wave-like equation case," Mathematical Models and Methods in Applied Sciences. In press.

[15] S. Ervedoza and E. Zuazua, "Uniformly exponentially stable approximations for a class of damped systems," Journal de Mathématiques Pures et Appliquées, vol. 91, no. 1, pp. 20-48, 2009. 


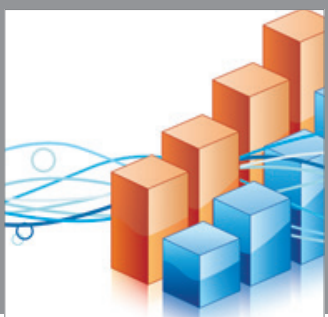

Advances in

Operations Research

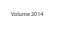

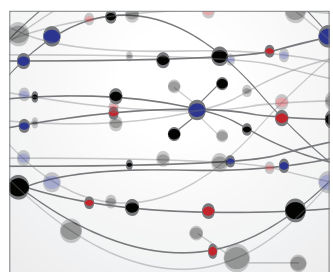

\section{The Scientific} World Journal
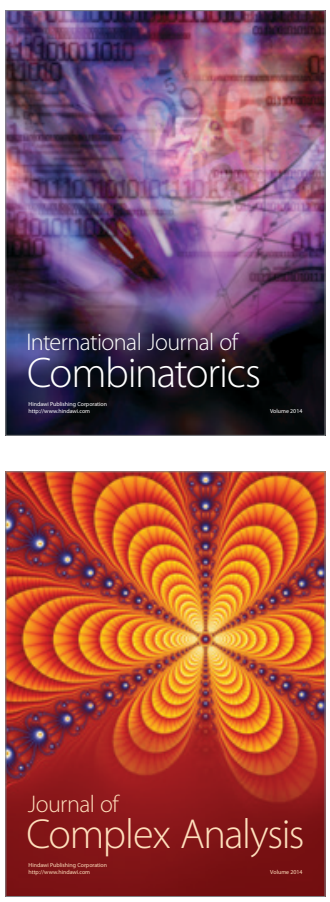

International Journal of

Mathematics and

Mathematical

Sciences
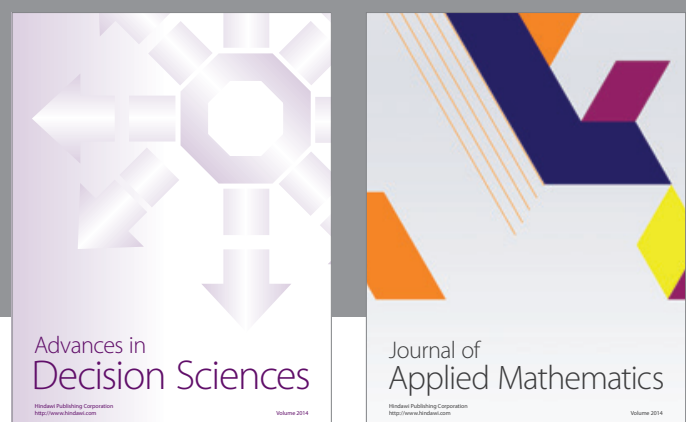

Journal of

Applied Mathematics
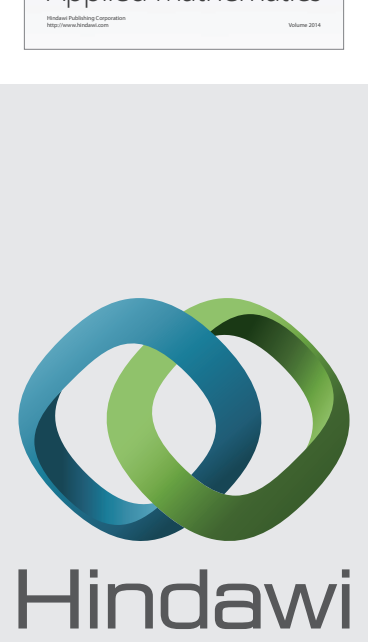

Submit your manuscripts at http://www.hindawi.com
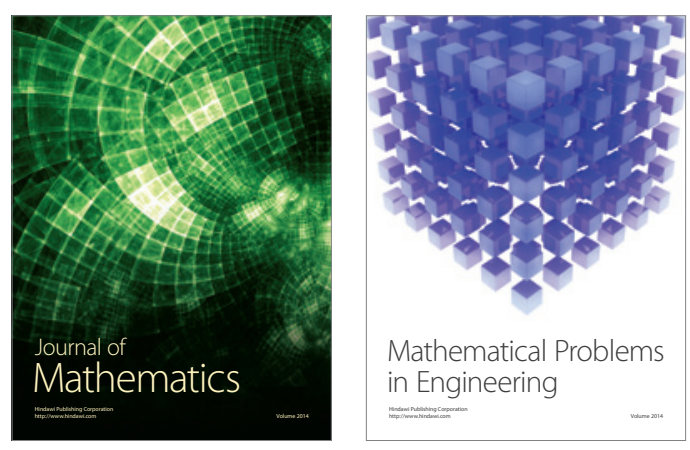

Mathematical Problems in Engineering
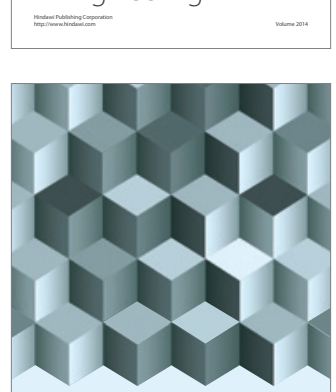

Journal of

Function Spaces
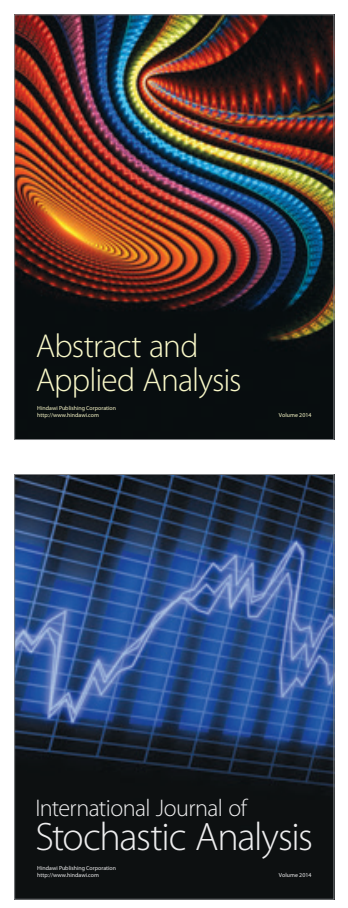

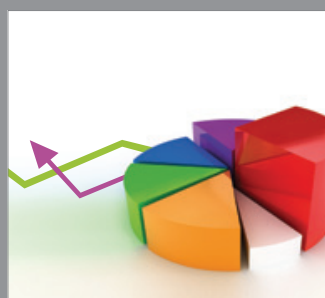

ournal of

Probability and Statistics

Promensencen
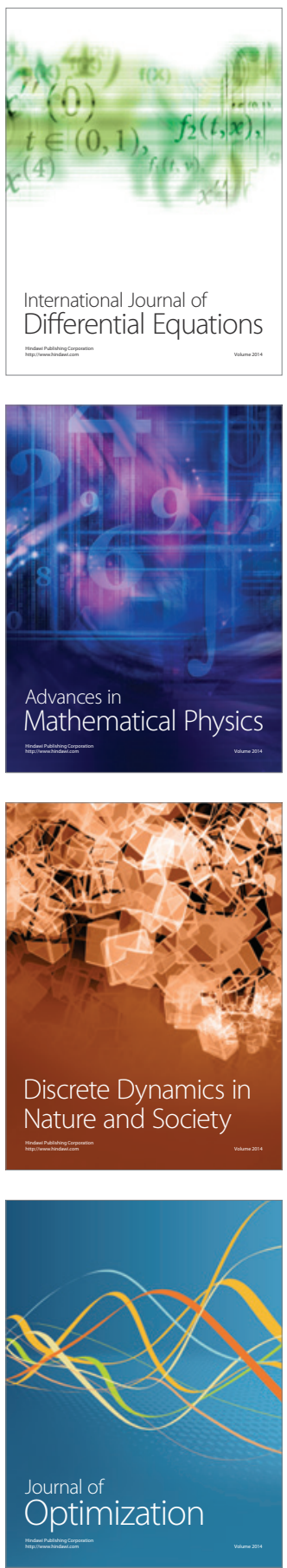